\title{
Femoral neck and bilateral scapular fractures in a 19-year-old male due to tetany. A case report
}

\author{
Alexander N. Mavrodontidis • George I. Mataliotakis • \\ Vasilios A. Kontogeorgakos • Dimitrios Pafilas • \\ Alexander E. Beris
}

Received: 17 October 2008 / Accepted: 27 October 2008 / Published online: 20 November 2008

(C) The Author(s) 2008. This article is published with open access at Springerlink.com

\begin{abstract}
Summary A case of simultaneous bilateral scapula fractures and a femoral neck fracture of the right hip suffered during hypocalcemic convulsions in a 19-year-old male is presented. The medical history, the treatment, and his latest follow-up are reported. The treatment approaches of such fractures in renal osteodystrophy patients are discussed.

Case report A case of simultaneous bilateral scapula fractures and a femoral neck fracture of the right hip suffered during hypocalcemic convulsions by a 19-year-old male is presented. His medical history, the treatment he received during the years following his first presentation as well as his latest follow-up 2 years later are reported.

Discussion The main issue of this report is the first osteosynthesis failure and the justification of the second. The treatment approaches of femoral neck fractures in patients with renal osteodystrophy are outlined, as they appear in the relevant literature.
\end{abstract}

Keywords Tetany · Hypocalcemic convulsions .

Osteopenic femoral neck fractures · Renal osteodystrophy .

Osteopenic fractures in uremic young adults

G. I. Mataliotakis $(\bowtie)$

George Papandreou Str 60C,

45444 Ioannina, Greece

e-mail: gimatas@yahoo.gr

A. N. Mavrodontidis $\cdot$ V. A. Kontogeorgakos $\cdot$ D. Pafilas $\cdot$

A. E. Beris

Orthopaedic Department, Ioannina University Hospital,

University Avenue,

45100 Ioannina, Greece

\section{Introduction}

The femoral neck is a biomechanically vulnerable area and therefore a common site of fractures when the bone is subjected to unusual loads as it is in the case of high energy trauma. Although the latter is the most common scenario, such unusual loads can be generated by excessive muscle contractions as well. This could happen during epileptic convulsions or during the course of electroshock therapy [1]. Certain medical conditions may further increase the possibility of such a rare fracture mechanism by diminishing bone strength. In patients suffering from renal osteodystrophy the combination of uremia and hypocalcemia can lead to bone demineralization and severe osteomalacia. At the same time hypocalcemic tetanic convulsions can expose bone to the above-mentioned unusual stresses resulting in pathologic fractures. The majority of these seizures occur during the dialysis sessions or in the immediate postoperative period following subtotal parathyroidectomy [1-5].

Although such events are rare, there are a few reports in the literature of patients suffering either bilateral scapula fractures or bilateral femoral neck fracture in the abovementioned settings [1-14]. However, we only came across one case of a patient having suffered both scapula and femoral neck fractures [3].

\section{Case report}

Having obtained the patient's full consent we present, in full detail, a case of a 19-year-old Caucasian male diagnosed with progressive renal failure at the age of 9 years. He had been treated with oral antiepileptic medication since early childhood. On a routine blood test a renal function compromise was discovered. As all other 
causes of renal failure where ruled out, oral carbamazepine was identified as the causative agent. It was immediately discontinued and no symptoms of epilepsy appeared ever since.

The patient's renal function was irreversibly damaged and chronic renal failure established. Renal biopsy revealed a histological picture of sclerotic glomerulonephritis. The patient has been on peritoneal dialysis ever since, as neither his legal guardians nor himself, as an adult, agreed to begin hemodialysis. Six years later he developed secondary anemia treated with subcutaneous erythropoietin, oral iron, and folic acid, secondary hyperparathyroidism treated with oral vitamin $\mathrm{D}$ and calcium supplements and chronic metabolic acidosis which required sodium bicarbonate supplementation. One year later, the patient developed secondary arterial hypertension controlled by proper oral antihypertensive medication. Eleven months later, the patient received a kidney transplant, his father being the donor. However the transplant soon failed due to renal vessel thrombosis. A second attempt was done after an interval of 1 month as a compatible donor was found. Unfortunately, the second renal transplant was also rejected.

Two and a half years later he underwent a subtotal parathyroidectomy. During the immediate postoperative period and despite intravenous calcium administration, the patient suffered an episode of generalized tetanic muscle contractions caused by a great drop in serum calcium levels. The convulsions were controlled with intravenous calcium gluconate and diazepam administration.

Before this episode the patient complained of occasional hip pain and had no ambulatory problems. Immediately after recovering from the generalized tetany episode the patient began complaining of severe pain in his right hip as well as in both shoulder areas. The X-rays revealed bilateral symmetrical transverse scapular fractures and a Garden IV, Pauwels II-III right femoral neck fracture (Fig. 1a,b). Since no other traumatic event took place, the severe muscle contractions were identified as the cause of the fractures.

The scapular fractures were treated conservatively with analgesics and upper limb motion restriction using a collar and cuff. The hip was immobilized with a skin traction device and the patient was put on prophylactic daily dose of LMWH as well as anti-osteoporotic medication and was scheduled for operative treatment. As the patient's general condition stabilized, 3 days later, and at the age of 19 years, he underwent a closed reduction and under fluoroscopic control, fracture stabilization with two cannulated Asnis Screws. On the third postoperative day he was allowed to walk on crutches not permitting weight bearing on the affected limb.

At 3-weeks post-op, radiological signs of loosening of the Asnis screws were obvious due to resorbtion at the fracture site. (Fig. 2a) The fracture failed to show signs of
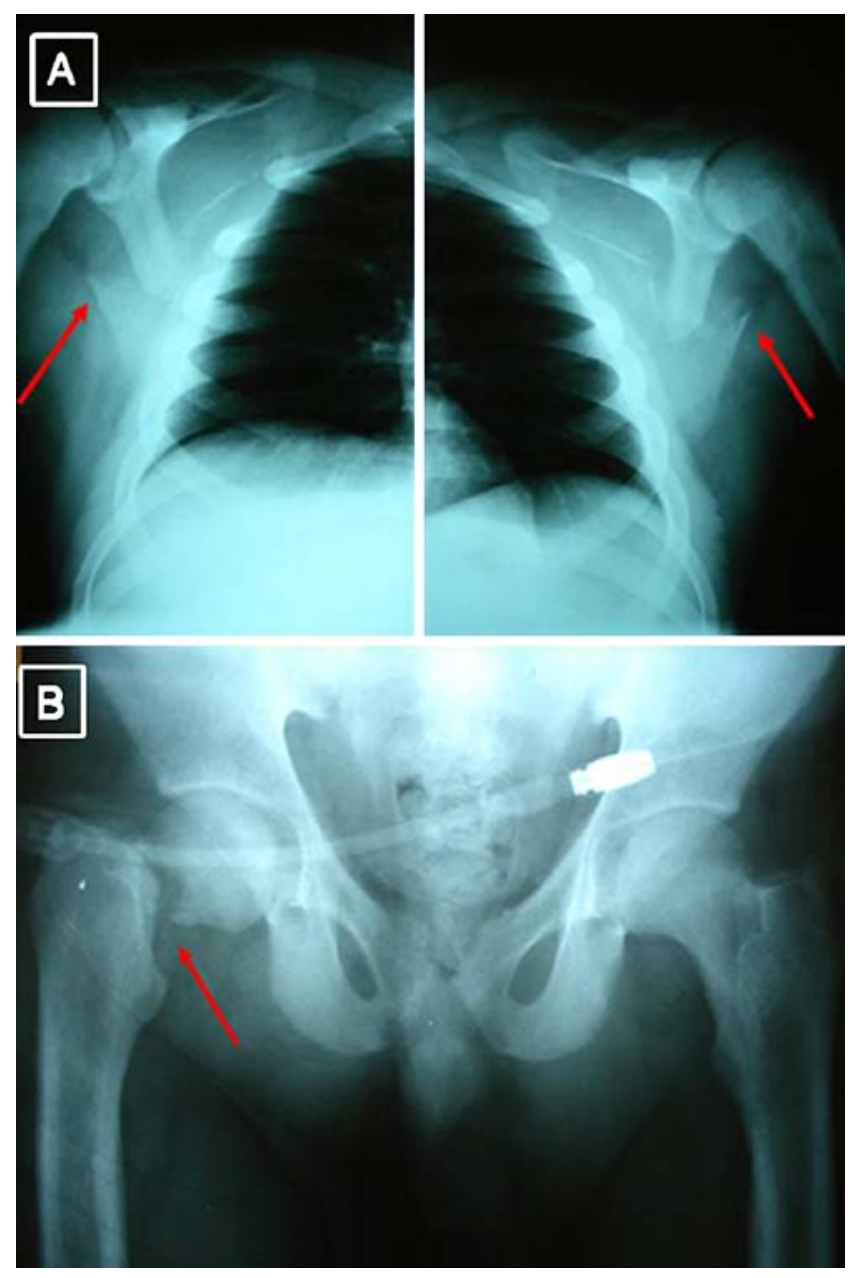

Fig. 1 a The arrow indicates the transcervical Garden IV, Pauwels IIIII right femoral neck fracture. The peritoneal lavage hose is evident. b The arrows indicate the bilateral scapular fractures

union on serial X-rays and ultimately resulted in non-union. At 20-weeks post-op, the screws had further loosened and cut out from the femoral head. (Fig. 2b) Furthermore, the femoral head was in varus displacement and its margins were sclerotic. However, since no radiological signs of necrosis of the femoral head were apparent, a revisional procedure of the fracture non-union was decided. After the Asnis screws were removed and fracture margins debrided, the femoral head was fixed in valgus orientation with a dynamic hip screw (DHS - Richards type) plus an antirotator cannulated Asnis Screw. (Fig. 2c)

Three months after the secondary procedure the fracture united and although the femoral head contour showed minor irregularities no significant radiological signs of osteonecrosis or osteoarthritis were apparent.

At his latest follow-up, 2 years after the secondary osteosynthesis procedure, the fracture has fully united with sufficient callus formation and without progression of valgus impaction. The screws are in place without any signs of loosening. The viability of femoral head shows no 

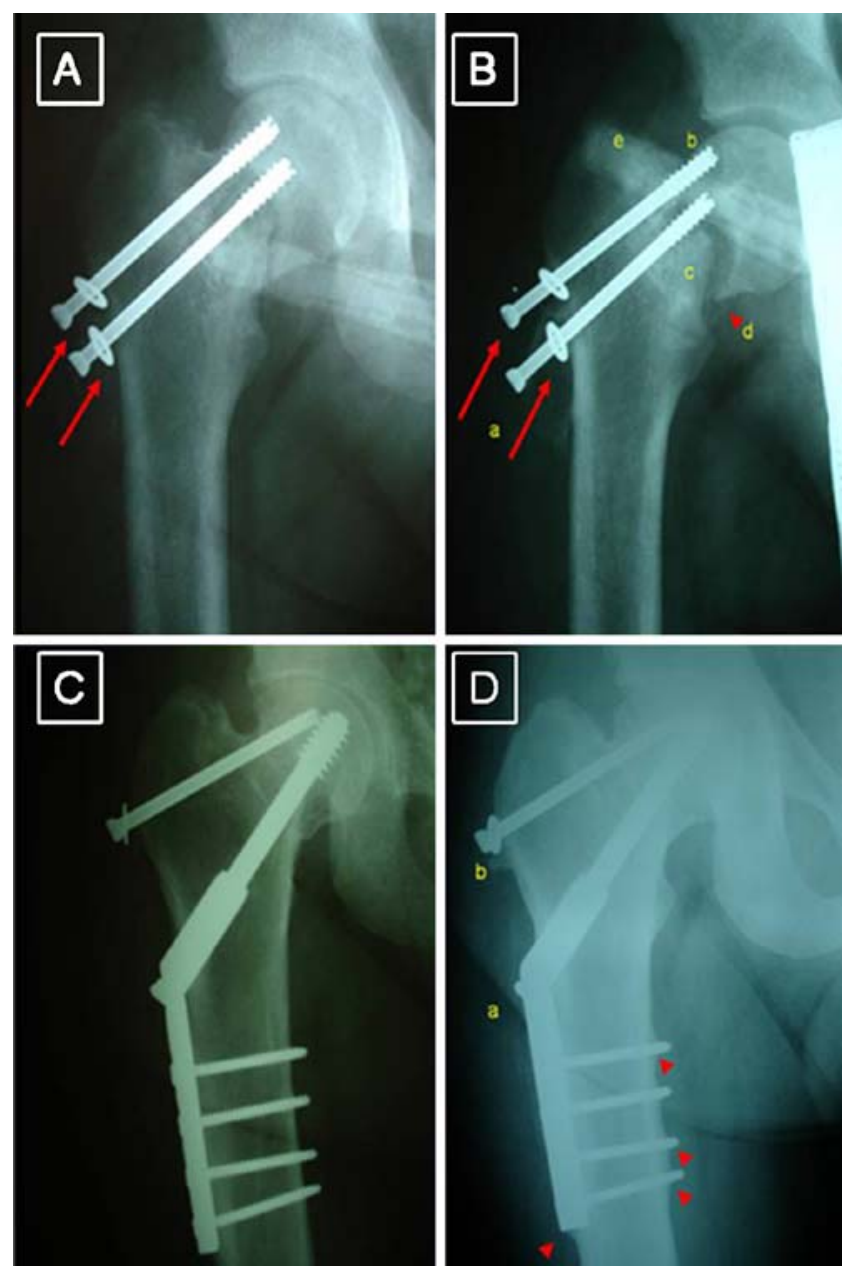

Fig. 2 a Three weeks post-op. Radiological signs of loosening of the Asnis screws are obvious due to resorbtion at the fracture site. b Twenty weeks post-op. The screws are further loosened (arrows, a) and cut out of the screws is obvious $(b)$. The femoral head is in varus displacement (arrowhead, $d$ ) and its margins were sclerotic (c). No radiological signs of necrosis of the femoral head are apparent. The peritoneal lavage hose projects on the major trochanter $(e)$. c Revisional procedure of the fracture non-union. The femoral head is fixed in valgus orientation with a dynamic hip screw (DHS-Richards type) plus an antirotator cannulated Asnis Screw. d The latest followup, 2 years after the secondary osteosynthesis procedure. The fracture is fully united with sufficient callus formation (arrowheads). No signs of malposition, progression of valgus impaction or deterioration are evident. The screws are in place without any signs of loosening $(a, b)$. The femoral head shows no radiological signs of deterioration or osteonecrosis

radiological signs of deterioration and the patient has no clinical signs of osteonecrosis. In fact he has an excellent Harris Hip Score and enjoys full outdoor activity (Fig. 2d).

\section{Discussion}

All scapular fractures, regardless their pattern, are conservatively treated by immobilization of the shoulder and upper limb [2-4]. Osteopenia resulting from secondary hyperparathyroidism and renal osteodystrophy make the hip vulnerable to fractures. The usually vertical pathological neck fractures - corresponding to type II or III according to Pauwels classification - are susceptible to pseudarthrosis (RockWood and Green's fractures of the neck of the femur, 1996), unless the indicated osteosynthesis is performed [15].

The general treatment regime for these fractures dictates internal fixation for all undisplaced or displaced fractures of the femoral head with good bone stock either by closed or open reduction if necessary. Preferred methods include cannulated screws, Knowles pins or the DHS with or without an antirotator screw $[2,16]$. In the case of older patients with underlying hip disease, cemented hemiarthroplasty or total hip arthroplasty, are strongly recommended (Orthopaedic Knowledge Update Trauma 2, 2000).

In this case report the patient was very young, with a low energy lesion and a normal life expectancy. He also was at the onset of his skeletal maturity with an obvious physeal plate but he presented with an adult-type lesion. The hemiarthroplasty was not a treatment option [1]. The least invasive procedure was the only possible way $[16,17]$. Since no recommendations were found in the literature on the treatment of these fractures and what kind of operative procedure to use [20], the first osteosynthesis was performed with two Asnis screws.

The BMD was lower as originally thought and the fracture collapsed. In osteopenic young patients with femoral neck fractures, a valgus osteotomy and internal fixation with either a DHS or an angular blade plate is recommended [16, 18].

Preservation of the femoral head is of utmost importance in young patients (Schatzker J. Subcapital and Intertrochanteric fractures), because osteonecrosis of the femoral head develops in $11-18 \%$ of all neck fractures in renal osteodystrophy $[5,19]$.

Removal of the old hardware and a revision osteosynthesis was decided. At first the antirotator Asnis Screw was placed and tightened to maintain the preferable valgus position of the femoral head. The second step was to place the sliding screw of the DHS to the proper position into the femoral head and to securely fix the plate on the femur.

During the after-treatment, young adults with such pathology and femoral neck fixated fractures should be prevented from weight bearing for a period of 12 weeks or longer (8-12 weeks in healthy individuals), depending on the radiographic assessment of the healing process [19]. At the same time a treatment regimen is planned aiming to regain normal or near-normal bone mass. This is accomplished by conservative means, such as oral nutritional supplements containing calcium, vitamin D and as well as other medication, such as biphosphonates. 
Our patient after the subtotal parathyroidectomy had his electrolyte balance corrected with the proper intravenous fluid administration, his diet contained the correct nutritional supplements and finally he received the above-mentioned progressive mobilization and physiotherapy.

Acknowledgements The authors thank their colleagues who have contributed to the combined treatment and follow-up of this patient. They also thank Theophanis Vasilakakos MD for his annotations at the final text preparation.

\section{Conflicts of interest None.}

Open Access This article is distributed under the terms of the Creative Commons Attribution Noncommercial License which permits any noncommercial use, distribution, and reproduction in any medium, provided the original author(s) and source are credited.

\section{References}

1. Taylor LJ, Grant SC (1985) Bilateral fracture of the femoral neck during a hypocalcaemic convulsion. A case report. J Bone Joint Surg Br 67(4):536-537

2. Gür S, Yilmaz H, Tüzüner S et al (1999) Fractures due to hypocalcemic convulsion. Int Orthop 23(5):308-309

3. Sakai S, David D, Shoji H et al (1976) Bone injuries due to tetany or convulsions during hemodialysis. Clin Orthop Relat Res 118:118-123

4. Peraino RA, Weinman EJ, Schloeder FX (1977) Unusual fractures during convulsions in two patients with renal osteodystrophy. South Med J 70(5):595-596

5. Madhok R, Rand JA (1993) Ten-year follow-up study of missed, simultaneous, bilateral femoral-neck fractures treated by bipolar arthroplasties in a patient with chronic renal failure. Clin Orthop Relat Res 291:185-187

6. Karapinar H, Ozdemir M, Akyol S et al (2003) Spontaneous bilateral femoral neck fractures in a young adult with chronic renal failure. Acta Orthop Belg 69(1):82-85
7. Hung KH, Lee CT, Gau YL et al (2001) Neglected bilateral femoral neck fractures in a patient with end-stage renal disease before chronic dialysis. Ren Fail 23(6):827-831

8. Zingraff J, Drueke T, Roux JP et al (1974) Bilateral fracture of the femoral neck complicating uremic bone disease prior to chronic hemodialysis. Clin Nephrol 2(2):73-75

9. Tarr RW, Kaye JJ, Nance EP Jr (1988) Insufficiency fractures of the femoral neck in association with chronic renal failure. South Med J 81(7):863-866

10. Undar L, Topcu S, Perçin S (1990) Simultaneous bilateral fractures of the femoral neck and superior pubis ramus following renal failure-induced hypocalcaemic convulsions. Br J Clin Pract 44(12):774-776

11. Schaab PC, Murphy G, Tzamaloukas AH et al (1990) Femoral neck fractures in patients receiving long-term dialysis. Clin Orthop Relat Res 260:224-231

12. Gerster JC, Charhon SA, Jaeger P et al (1983) Bilateral fractures of femoral neck in patients with moderate renal failure receiving fluoride for spinal osteoporosis. Br Med J (Clin Res Ed) 287 (6394):723-725 Sep 10

13. Bednarek-Skublewska A, Kołodziej R, Baranowicz-Gaszczyk I et al (2003) Femoral neck fractures in hemodialysis patients. Przegl Lek 60(11):682-685

14. Kazimoğlu C, Yağdi S, Karapinar $\mathrm{H}$ et al (2007) Bilateral quadriceps tendon rupture and coexistent femoral neck fracture in a patient with chronic renal failure. Acta Orthop Traumatol Turc 41(5):393-396

15. Evarts CM, Pauwels F (1990) Classification of femoral neck fractures. In: Surgery of the musculoskeletal system (1990), 2nd edn. Churchill Livingstone, New York, p 2556

16. Swiontkowski MF (2003) Intracapsular Hip Fractures. In: Browner B, Jupiter J, Levine A, Trafton P (eds) Skeletal Trauma, 3rd edn. Saunders $\mathrm{p}$ 1714-1732

17. Giannoudis PV, Schneider E (2006) Principles of fixation in osteoporotic fractures. J Bone Joint Surg Br 88(10):1272-1278. Review

18. Magu NK, Singh R, Sharma A (2005) Treatment of pathologic femoral neck fractures with modified Pauwels' osteotomy. Clin Orthop Relat Res 437:229-235

19. Bonnaire F, Zenker H, Lill C et al (2005) Treatment strategies for proximal femur fractures in osteoporotic patients. Osteoporos Int 16(Suppl 2):S93-S102. Epub 2004 Oct 16. Review

20. Chadha M, Balain B, Maini L et al (2001) Spontaneous bilateral displaced femoral neck fractures in nutritional osteomalacia-a case report. Acta Orthop Scand 72(1):94-96 